\title{
Estimates of singular integrals and multilinear commutators in weighted Morrey spaces
}

\section{Xiao Feng Ye* and Xu Sheng Zhu}

\section{"Correspondence:}

mathyxf@yahoo.cn;

xiaofye@ecjtu.jx.cn

Department of Mathematics and

Information Sciences, East China

Jiao Tong University, Nanchang,

330013, P.R. China

\begin{abstract}
Suppose $T$ is a singular integral operator whose kernel is a variable kernel with mixed homogeneity; the purpose of this paper is to study the continuity of the operator in weighted Morrey spaces $L^{p, \kappa}(\omega), 1 \leq p<\infty, 0<\kappa<1$. A special attention is paid also to the multilinear commutator of this operator with $B M O$ function.
\end{abstract}

MSC: 42B20; 42B35

Keywords: mixed homogeneity; multilinear commutators; weighted Morrey spaces; $B M O$; Orlicz maximal operator

\section{Introduction}

Let $K(x, \xi): \mathbb{R}^{n} \times \mathbb{R}^{n} \backslash\{0\} \rightarrow \mathbb{R}$ be a variable kernel. The singular integral operator is defined by

$$
T f(x)=p \cdot v \cdot \int_{\mathbb{R}^{n}} K(x, x-y) f(y) d y
$$

and its multilinear commutator with the $B M O$ function

$$
[\vec{b}, T] f(x)=\int_{\mathbb{R}^{n}} \prod_{i=1}^{N}\left(b_{i}(x)-b_{i}(y)\right) K(x, x-y) f(y) d y,
$$

where $\vec{b}=\left(b_{1}, \ldots, b_{n}\right), b_{i} \in B M O, 1 \leq i \leq N$. The variable kernel $K(x, \xi)$ depends on some parameter $x$ and possesses 'good' properties with respect to the second variable $\xi$, which was firstly introduced by Fabes and Rieviève in [1]. They generalized the classical Calderón and Zygmund kernel and the parabolic kernel studied by Jones in [2]. By introducing a new metric $\rho$, Fabes and Rieviève studied (1.1) in $L^{p}\left(\mathbb{R}^{n}\right)$, where $\mathbb{R}^{n}$ was endowed with the topology induced by $\rho$ and defined by ellipsoids.

By using this metric $\rho$, Softova in [3] obtained that the integral operator (1.1) and commutator were continuous in generalized Morrey spaces $L^{p, \omega}\left(\mathbb{R}^{n}\right), 1<p<\infty, \omega$ satisfying suitable conditions.

The multilinear commutator was introduced by Pérez and González [4] who proved the weighted Lebesgue estimates. Xu in [5] also showed that the multilinear commutators (1.2) were continuous in $L^{p, \omega}\left(\mathbb{R}^{n}\right), 1<p<\infty$.

The weighted Morrey spaces $L^{p, \kappa}(w)$ were introduced by Komori and Shirai [6]. Moreover, they showed some classical integral operators and corresponding commutators were

() 2012 Ye and Zhu; licensee Springer. This is an Open Access article distributed under the terms of the Creative Commons Attribution License (http://creativecommons.org/licenses/by/2.0), which permits unrestricted use, distribution, and reproduction in any medium, provided the original work is properly cited. 
bounded in weighted Morrey spaces. Recently, Wang [7-9] obtained that some other kind of integral operators (e.g., Bochner-Riesz operator, Marcinkiewicz operators etc.) and commutators were also bounded in weighted Morrey spaces. He Sha [10] showed that multilinear operators were bounded on weighted Morrey spaces with the symbol of $b \in \operatorname{Lip}(\beta)$. The main purpose of this paper is to discuss the continuity of the singular integral operator whose kernel is a variable kernel with mixed homogeneity and its multilinear commutator in the weighted Morrey spaces $L^{p, \kappa}(\omega), 1<p<\infty, 0<\kappa<1$, where the weight function $\omega$ is $A_{p}$ weight. Furthermore, we shall give the weighted weak type estimate of theses operators in the weighted Morrey spaces $L^{1, \kappa}(\omega), 0<\kappa<1$. Our main results are stated as follows.

Theorem 1.1 Let $1<p<\infty, 0<\kappa<1$. If $w \in A_{p}$, then there exists a constant $C>0$ such that

$$
\|T f\|_{L^{p, \kappa}(w)} \leq C\|f\|_{L^{p, \kappa}(w)} .
$$

When $p=1$, for any $\lambda>0$ and ellipsoid $\mathcal{E}$, there exists a constant $C>0$ such that

$$
\lambda w(\{x \in \mathcal{E}:|T f(x)|>\lambda\}) \leq C\|f\|_{L^{1, k}(w)} .
$$

If $K(x, \xi)$ is a constant kernel and a metric $\rho$ is Euclidean one, this result is just Theorem 3.3 in [6].

Theorem 1.2 Let $1<p<\infty, 0<\kappa<1$. If $b_{i} \in B M O\left(\mathbb{R}^{n}\right), 1 \leq i \leq N, w \in A_{p}$, then there exists a constant $C>0$ such that

$$
\|[\vec{b}, T] f\|_{L^{p, \kappa}(w)} \leq C\|\vec{b}\|\|f\|_{L^{p, \kappa}(w)}
$$

where $\|\vec{b}\|=\prod_{i=1}^{N}\left\|b_{i}\right\|_{*}$. When $p=1$, for any $\lambda>0$ and ellipsoid $\mathcal{E}$, then there exists a constant $C>0$ such that

$$
\lambda w(\{x \in \mathcal{E}:|[\vec{b}, T] f(x)|>\lambda\}) \leq C\|\vec{b}\|\|f\|_{L^{\Phi, k}(w)},
$$

where $\Phi(t)=t \log ^{N}(e+t)$ and $\|f\|_{L^{\Phi, \kappa}(w)}=\|\Phi(|f|)\|_{L^{1, \kappa}(w)}$.

In what follows, we denote by $C$ positive constants which are independent of the main parameters but may vary from line to line.

\section{Some notations and lemmas}

In this section, we introduce some basic definitions and lemmas needed for the proof of the main results.

Let $\alpha_{1}, \ldots, \alpha_{n}$ be real numbers, $\alpha_{i} \geq 1$ and $|\alpha|=\sum_{i=1}^{n} \alpha_{i}$. Following Fabes and Riviève [1], there exists a function $\rho$ such that $\rho(x-y)$ defines a distance between any two points $x, y \in \mathbb{R}^{n}$. Thus $\mathbb{R}^{n}$ endowed with the metric $\rho$ results in a homogeneous metric space $[1,3]$. The balls with respect to $\rho(x)$ centered at the origin and of radius $\mathrm{r}$ are the ellipsoids

$$
\mathcal{E}_{r}(0)=\left\{x \in \mathbb{R}^{n}: \frac{x_{1}^{2}}{r^{2 \alpha_{1}}}+\cdots+\frac{x_{n}^{2}}{r^{2 \alpha_{n}}}<1\right\}
$$


with Lebesgue measure $\left|\mathcal{E}_{r}\right|=C(n) r^{|\alpha|}$. It is easy to see that the unit sphere with respect to this metric coincides with the unit sphere $\Sigma_{n}$ with respect to the Euclidean one.

Definition 2.1 The function $K(x, \xi): \mathbb{R}^{n} \times \mathbb{R}^{n} \backslash\{0\} \rightarrow \mathbb{R}$ is called a variable kernel with mixed homogeneity if

(i) for every fixed $x$, the function $K(x, \cdot)$ is a constant kernel satisfying

(1) $K(x, \cdot) \in C^{\infty}\left(\mathbb{R}^{n} \backslash\{0\}\right)$;

(2) for any $\mu>0, \alpha_{i} \geq 1,|\alpha|=\sum_{i=1}^{n} \alpha_{i}$

$$
K\left(x, \mu^{\alpha_{1}} \xi_{1}, \ldots, \mu^{\alpha_{n}} \xi_{n}\right)=\mu^{-|\alpha|} K(x, \xi) ;
$$

(3) $\int_{\Sigma_{n}} K(x, \xi) d \sigma_{\xi}=0$ and $\int_{\Sigma_{n}}|K(x, \xi)| d \sigma_{\xi}<\infty$;

(ii) for every multiindex $\beta, \sup _{\xi \in \Sigma_{n}}\left|D_{\xi}^{\beta} K(x, \xi)\right| \leq C(\beta)$ independent of $x$.

In the case $\alpha_{i}=1,1 \leq i \leq n$, Definition 2.1 gives rise to the classical Calderón-Zygmund kernel. On the other hand, when $\alpha_{i}=1,1 \leq i \leq n-1$ and $\alpha_{n} \geq 1$, we obtain the kernel studied by Jones in [2] and discussed in [1].

Definition 2.2 Let $1 \leq p<\infty, 0<\kappa<1$ and $w$ be a weight function. Then a weighted Morrey space is defined by

$$
L^{p, \kappa}(w):=\left\{f \in L_{\mathrm{loc}}^{1}(w):\|f\|_{L^{p, \kappa}(w)}<\infty\right\},
$$

where

$$
\|f\|_{L^{p, \kappa}(w)}=\sup _{\mathcal{E}}\left(\frac{1}{w(\mathcal{E})^{\kappa}} \int_{\mathcal{E}}|f(x)|^{p} w(x) d x\right)^{1 / p},
$$

the supremum is taken over all ellipsoid $\mathcal{E}$ in $\mathbb{R}^{n}$.

Definition 2.3 For the function $b \in L_{\mathrm{loc}}^{1}\left(\mathbb{R}^{n}\right)$ and any ellipsoid $\mathcal{E}, b$ is called a $B M O$ function if

$$
\|b\|_{*}=\sup _{\mathcal{E}} \frac{1}{|\mathcal{E}|} \int_{\mathcal{E}}\left|b(x)-b_{\mathcal{E}}\right| d x<\infty
$$

where $b_{\mathcal{E}}=\frac{1}{|\mathcal{E}|} \int_{\mathcal{E}} b(y) d y$. The quantity $\|b\|_{*}$ is a norm in the $B M O$ modulo constant function under which $B M O$ results in a Banach space (see [11]).

Definition 2.4 Let $1<p<\infty$. For any locally integrable function $w$ and ellipsoid $\mathcal{E}$, if

$$
\left(\frac{1}{|\mathcal{E}|} \int_{\mathcal{E}} w(x) d x\right)\left(\frac{1}{|\mathcal{E}|} \int_{\mathcal{E}} w(x)^{\frac{1}{1-p}} d x\right)^{p-1}<\infty
$$

holds, then $w$ belongs to the Muckenhoupt class $A_{p}$. We denote $A_{\infty}=\bigcup_{1<p<\infty} A_{p}$.

When $p=1, w \in A_{1}$ if there exists $C>1$ such that

$$
M w(x) \leq C w(x)
$$

for almost every $x \in \mathbb{R}^{n}$. 
Remark 2.5 Given a weight function $w \in A_{p}, 1 \leq p \leq \infty$, it also satisfies the doubling condition $\Delta_{2}$ : for any ellipsoid $\mathcal{E}$, there exists a constant $C>0$ such that $w(2 \mathcal{E}) \leq C w(\mathcal{E})$.

In fact, $w \in \Delta_{2}$, we have the following inequality.

Lemma A $[6,12]$ Suppose $w \in \Delta_{2}$, there exists a constant $D>1$ such that

$$
w(2 \mathcal{E}) \geq D w(\mathcal{E})
$$

for any ellipsoid $\mathcal{E}$.

Lemma B [13] Suppose $w \in A_{\infty}$, then the norm of $B M O(w)$ is equivalent to the norm of $B M O\left(\mathbb{R}^{n}\right)$, where

$$
B M O(w)=\left\{b:\|b\|_{*, w}=\sup _{\mathcal{E}} \frac{1}{w(\mathcal{E})} \int_{\mathcal{E}}\left|b(x)-b_{\mathcal{E}, w}\right| w(x) d x<\infty\right\},
$$

where $b_{\mathcal{E}, w}=\frac{1}{w(\mathcal{E})} \int_{\mathcal{E}} b(x) w(x) d x$.

Lemma $\mathbf{C}$ [14] Let the ellipsoid $\mathcal{E}=\mathcal{E}\left(x_{0}, r\right)$ centered at $x_{0}$ with side length of $r$. For any positive integer $i, 2^{i} \mathcal{E}$ denotes the ellipsoid centered at $x_{0}$ with side length of $2^{i} r$, we have the inequality

$$
\left|b_{2^{i} \mathcal{E}}-b_{\mathcal{E}}\right| \leq C i\|b\|_{*}
$$

where $b_{\mathcal{E}, w}=\frac{1}{|\mathcal{E}|} \int_{\mathcal{E}} b(x) w(x) d x$

Lemma D [6] Suppose $1<p<\infty, 0<\kappa<1$ and $w \in A_{p}$, if $\bar{T}$ is the classical CalderónZygmund operator with a constant kernel, then the operator $\bar{T}$ is bounded on $L^{p, \kappa}(w)$.

If $p=1,0<\kappa<1$ and $w \in A_{1}$, then there exists a constant $C>0$ such that

$$
\lambda w(\{x \in \mathcal{E}:|\bar{T} f(x)|>\lambda\}) \leq C\|f\|_{L^{1, \kappa}(w)} w(\mathcal{E})^{\kappa}
$$

for all $\lambda>0$ and any ellipsoid $\mathcal{E}$.

Definition 2.6 Let $\Phi(t)=t \log ^{N}(t+e)$. The Orlicz maximal operator $M_{\Phi}$ is given by

$$
M_{\Phi} f(x)=\sup _{x \in \mathcal{E}}\|f\|_{\Phi, \mathcal{E}}=\sup _{x \in \mathcal{E}} \frac{1}{|\mathcal{E}|} \int_{\mathcal{E}} \Phi(|f|)(x) d x .
$$

From the above definition, observe that $M f(x) \leq M_{\Phi} f(x) \leq M(\Phi(|f|))(x)$. This inequality will be relevant in our work.

Aside from the properties of an $A_{p}$ weight function and a $B M O$ function, we need some estimates of multilinear commutators. The following results were proved by Pérez and González [4].

Lemma $\mathbf{E}$ Let $1<p<\infty$ and $w \in A_{p}$. Suppose $b_{j} \in B M O\left(\mathbb{R}^{n}\right), 1 \leq j \leq N$, then there exists a constant $C>0$ such that

$$
\int_{\mathbb{R}^{n}}|[\vec{b}, \bar{T}](f)(x)|^{p} w(x) d x \leq C\|\vec{b}\|^{p} \int_{\mathbb{R}^{n}}|f(x)|^{p} w(x) d x .
$$


Although the commutators with a $B M O$ function are not of weak type $(1,1)$, we have the following inequality.

Lemma $\mathbf{F}$ Let $w \in A_{\infty}$. There exists a constant $C>0$ such that

$$
\begin{aligned}
& \sup _{t>0} \frac{1}{\Phi\left(\frac{1}{t}\right)} w\left(x \in \mathbb{R}^{n}:|[\vec{b}, \bar{T}](f)(x)|>t\right) \\
& \quad \leq C \sup _{t>0} \frac{1}{\Phi\left(\frac{1}{t}\right)} w\left(x \in \mathbb{R}^{n}: M_{\Phi}(\|\vec{b}\| f)(x)>t\right),
\end{aligned}
$$

where $\Phi(t)=t \log ^{N}(e+t)$.

By the above inequality, we have the following result.

Lemma G Let $w \in A_{1}$. There exists a constant $C>0$ such that, for all $\lambda>0$,

$$
w\left(x \in \mathbb{R}^{n}:|[\vec{b}, \bar{T}](f)(x)|>\lambda\right) \leq C \int_{\mathbb{R}^{n}} \Phi(|f|)(x) w(x) d x,
$$

where $\Phi(t)=t \log ^{N}(e+t)$.

Finally, we need the spherical harmonics and their properties (see more detail in $[1,15,16])$. Recall that any homogeneous polynomial $P: \mathbb{R}^{n} \rightarrow \mathbb{R}$ of degree $m$ that satisfies $\Delta P=0$ is called an $n$-dimensional solid harmonic of degree $m$. Its restriction to the unit sphere $\Sigma_{n}$ will be called an $n$-dimensional spherical harmonic of degree m. Denote by $H_{m}$ the space of all $n$-dimensional spherical harmonics of degree $m$. In general, it results in a finite-dimensional linear space with $g_{m}=\operatorname{dim} H_{m}$ such that $g_{0}=1, g_{1}=n$ and

$$
g_{m}=C_{m+n-1}^{n-1}-C_{m+n-3}^{n-1} \leq C(n) m^{n-2}, \quad m \geq 2 .
$$

Furthermore, let $\left\{Y_{s m}\right\}_{s=1}^{g_{m}}$ be an orthonormal basis of $H_{m}$. Then $\left\{Y_{s m}\right\}_{s=1 m=0}^{g_{m} \infty}$ is a complete orthonormal system in $L^{2}\left(\Sigma_{n}\right)$ and

$$
\sup _{x \in \Sigma_{n}}\left|D_{x}^{\beta} Y_{s m}(x)\right| \leq C(n) m^{|\beta|+(n-2) / 2}, \quad m=1,2, \ldots
$$

If, for instance, $\phi \in C^{\infty}\left(\Sigma_{n}\right)$, then $\Sigma_{s, m} b_{s m} Y_{s m}(x)$ is the Fourier series expansion of $\phi(x)$ with respect to $\left\{Y_{s m}\right\}_{s, m}\left(\Sigma_{s, m}\right.$ substitutes $\left.\Sigma_{m=0}^{\infty} \Sigma_{s=1}^{g_{m}}\right)$ and

$$
b_{s m}=\int_{\Sigma_{n}} \phi(x) Y_{s m}(x) d \sigma, \quad\left|b_{s m}\right| \leq C(n, l) m^{-2 l} \sup _{\substack{|\beta|=2 l \\ y \in \Sigma_{n}}}\left|D_{y}^{\beta} \phi(y)\right| \text {, }
$$

for any integer $l$. In particular, the expansion of $\phi$ into spherical harmonics converges uniformly to $\phi$. For more detail, we can see [15].

\section{Proof of the theorems}

In this section, we shall use the complete orthonormal system in $L^{2}\left(\Sigma_{n}\right)$ and some lemmas as above to finish the theorems. 
Proof of Theorem 1.1 In order to ensure the existence of the operator (1.1) in $L^{p, \kappa}(w), 1 \leq$ $p<\infty$, we restrict our consideration to the function $f \in L^{p, \kappa}(w)$, for which the norm of $L^{p}(w)$ is finite. For the sake of convenience, we still denote these spaces by $L^{p, \kappa}(w)$. Let $x, y \in \mathbb{R}^{n}$ and $\bar{y}=y / \rho(y) \in \Sigma_{n}$. In view of the properties of the kernel $K$ with respect to the second variable and the complete of $\left\{Y_{s m}(x)\right\}$ in $L^{2}\left(\Sigma_{n}\right)$, we get

$$
\begin{aligned}
K(x, x-y) & =\rho(x-y)^{-|\alpha|} K(x, \overline{x-y}) \\
& =\rho(x-y)^{-|\alpha|} \sum_{s, m} b_{s m}(x) Y_{s m}(\overline{x-y}) .
\end{aligned}
$$

Replacing the kernel with its series expansion, (1.1) can be written as

$$
\begin{aligned}
T f(x) & =\lim _{\epsilon \rightarrow 0} T_{\epsilon} f(x) \\
& =\lim _{\epsilon \rightarrow 0} \int_{\rho(x-y)>\epsilon} \sum_{s, m} b_{s m}(x) \rho(x-y)^{-|\alpha|} Y_{s m}(\overline{x-y}) f(y) d y .
\end{aligned}
$$

From the properties of (2.1)-(2.3), the series expansion $\sum_{s, m}\left|b_{s m}(x) Y_{s m}(\overline{x-y})\right| \leq$ $C(n, \alpha) m^{3(n-2) / 2-2 l}$, where the integer $l$ is preliminarily chosen greater than $(3 n-4) / 4$. Along with the $\rho(x-y)^{-|\alpha|} f(y) \in L^{1}\left(\mathbb{R}^{n}\right)$ for a.a. $x \in \mathbb{R}^{n}$, by the Fubini dominated convergence theorem, we have

$$
T f(x)=\sum_{s, m} b_{s m}(x) \lim _{\epsilon \rightarrow 0} \int_{\rho(x-y)>\epsilon} H_{s m}(x-y) f(y) d y=\sum_{s, m} b_{s m}(x) T_{s m} f(x),
$$

where $H_{s m}(x-y)=\rho(x-y)^{-|\alpha|} Y_{s m}(\overline{x-y})$. Instead of the operators $T f(x)$, we shall study the existence and boundedness in $L^{p, \kappa}(\omega)$ of the operators $T_{s m} f(x)$ with a kernel $H_{s m}(\cdot)$. Observe that $H_{s m}(\cdot)$ is a constant kernel and satisfies

$$
\left|H_{s m}(x)\right| \leq C(n, \alpha) m^{\frac{n-2}{2}} \rho^{-|\alpha|} ; \quad\left|\nabla H_{s m}(x)\right| \leq C(n, \alpha) m^{\frac{n}{2}} \rho^{-|\alpha|-1} .
$$

From Lemma D, it follows

$$
\left\|T_{s m} f(x)\right\|_{L^{p, \kappa}(\omega)} \leq C(n, \alpha) m^{\frac{n}{2}}\|f(x)\|_{L^{p, \kappa}(\omega)}
$$

for $1<p<\infty$. Consequently, by the above inequality and (2.1)-(2.3), we show

$$
\begin{aligned}
& \|T f(x)\|_{L^{p, \kappa}(\omega)} \leq C \sum_{s, m}\left\|b_{s m}(x)\right\|_{L^{\infty}}\left\|T_{s m} f(x)\right\|_{L^{p, \kappa}(\omega)} \\
& \leq C \sum_{s, m} m^{-2 l+\frac{n}{2}}\|f(x)\|_{L^{p, \kappa}(\omega)} \\
& \leq C\|f\|_{L^{p, \kappa}(\omega)},
\end{aligned}
$$

where the integer $l$ is preliminary chosen greater that $l>\frac{3 n}{4}$. For $p=1$, by Lemma $\mathrm{D}$, we have

$$
\lambda w\left(\left\{x \in \mathcal{E}:\left|T_{s m} f(x)\right|>\lambda\right\}\right) \leq C(n, \alpha) m^{\frac{n}{2}}\|f\|_{L^{1, \kappa}(w)}
$$


for any $\lambda>0$ and ellipsoid $\mathcal{E}$. Therefore, one gets

$$
\begin{aligned}
\lambda w(\{x \in \mathcal{E}:|T f(x)|>\lambda\}) & \leq C \sum_{s, m}\left\|b_{s m}(x)\right\|_{L^{\infty}} \lambda w\left(\left\{x \in \mathcal{E}:\left|T_{s m} f(x)\right|>\lambda\right\}\right) \\
& \leq C \sum_{s, m} m^{-2 l+\frac{n}{2}}\|f\|_{L^{1, \kappa}(w)} \\
& \leq C\|f(x)\|_{L^{1, \kappa}(w)}
\end{aligned}
$$

thus we complete the proof of Theorem 1.1.

Next we begin with the second theorem, for which further discussion is needed.

Proof of Theorem 1.2 As above, we use the series expansion of a kernel $K(x, y)$, the operator $[\vec{b}, T] f(x)$ is divided into

$$
[\vec{b}, T] f(x)=\sum_{s, m} b_{s m}(x)\left[\vec{b}, T_{s m}\right] f(x)
$$

Instead of the operator $[\vec{b}, T] f(x)$, we only consider the existence and boundedness in $L^{p, \kappa}(w)$ of the operators $\left[\vec{b}, T_{s m}\right] f(x)$.

Let $1<p<\infty$. For any ellipsoid $\mathcal{E}$, we only need to obtain the inequality

$$
\int_{\mathcal{E}}\left|\left[\vec{b}, T_{s m}\right] f(x)\right|^{p} w(x) d x \leq C m^{\frac{m p}{2}}\|b\|^{p} w(\mathcal{E})^{k}\|f\|_{L^{p, k}(w)}^{p} .
$$

In fact, by the series expansion of a kernel $K(x, y)$, we have

$$
\begin{aligned}
\|[\vec{b}, T] f(x)\|_{L^{p, \kappa}(\omega)} & \leq C \sum_{s, m}\left\|b_{s m}(x)\right\|_{L^{\infty}}\left\|\left[\vec{b}, T_{s m}\right] f(x)\right\|_{L^{p, \kappa}(\omega)} \\
& \leq C \sum_{s, m} m^{-2 l+\frac{n}{2}}\|f\|_{L^{p, \kappa}(\omega)} \leq C\|f\|_{L^{p, \kappa}(\omega)}
\end{aligned}
$$

where the integer $l$ is chosen greater than $l>\frac{3 n}{4}$. Next, fix the above ellipsoid $\mathcal{E}=\mathcal{E}\left(x_{0}, r\right)$ and decompose $f=f_{1}+f_{2}$, where $f_{1}=f \chi_{2 \mathcal{E}}, \chi_{2 \mathcal{E}}$ denotes the characteristic function of $2 \mathcal{E}$, then we have

$$
\begin{aligned}
\int_{\mathcal{E}}\left|\left[\vec{b}, T_{s m}\right](f)(x)\right|^{p} w(x) d x & \leq C \int_{\mathcal{E}}\left\{\left|\left[\vec{b}, T_{s m}\right]\left(f_{1}\right)(x)\right|^{p}+\left|\left[\vec{b}, T_{s m}\right]\left(f_{2}\right)(x)\right|^{p}\right\} w(x) d x \\
& =C\{I+I I\} .
\end{aligned}
$$

By using Lemma E, we get

$$
\begin{aligned}
I & \leq \int_{\mathbb{R}^{n}}\left|\left[\vec{b}, T_{s m}\right]\left(f_{1}\right)(x)\right|^{p} w(x) d x \\
& \leq C m^{\frac{n p}{2}}\|\vec{b}\|^{p} w(\mathcal{E})^{\kappa}\|f\|_{L^{p, \kappa}(w)}^{p} .
\end{aligned}
$$


For the term $I I$, without loss of generality, we can assume $N=2$. Thus, the operator $\left[\vec{b}, T_{s m}\right]$ can be divided into four parts,

$$
\begin{aligned}
{\left[\vec{b}, T_{s m}\right] f_{2}(x)=} & \left(b_{1}(x)-\lambda_{1}\right)\left(b_{2}(x)-\lambda_{2}\right) \int_{\mathbb{R}^{n}} H_{s m}(x-y) f_{2}(y) d y \\
& +\int_{\mathbb{R}^{n}} H_{s m}(x-y)\left(b_{1}(y)-\lambda_{1}\right)\left(b_{2}(y)-\lambda_{2}\right) f_{2}(y) d y \\
& -\left(b_{1}(x)-\lambda_{1}\right) \int_{\mathbb{R}^{n}} H_{s m}(x-y)\left(b_{2}(y)-\lambda_{2}\right) f_{2}(y) d y \\
& -\left(b_{2}(x)-\lambda_{2}\right) \int_{\mathbb{R}^{n}} H_{s m}(x-y)\left(b_{1}(y)-\lambda_{1}\right) f_{2}(y) d y \\
= & I I_{1}(x)+I I_{2}(x)+I I_{3}(x)+I I_{4}(x),
\end{aligned}
$$

where $\lambda_{i}=\left(b_{i}\right)_{\mathcal{E}, w}=\frac{1}{w(\mathcal{E})} \int_{\mathcal{E}} b_{i}(x) w(x) d x, i=1$, 2. For the term $I I_{1}(x)$, observing that $x \in \mathcal{E}$ and $y \in \mathbb{R}^{n} \backslash 2 \mathcal{E}$, we have $\rho\left(x_{0}-y\right) \leq C \rho(x-y)$. Thus, it yields

$$
\begin{aligned}
\int_{\mathcal{E}}\left|I I_{1}(x)\right|^{p} w(x) d x \leq & C m^{\frac{n p}{2}} \int_{\mathcal{E}}\left|\left(b_{1}(x)-\left(b_{1}\right)_{\mathcal{E}, w}\right)\left(b_{2}(x)-\left(b_{2}\right)_{\mathcal{E}, w}\right)\right|^{p} w(x) d x \\
& \times\left(\int_{\mathbb{R}^{n} \backslash 2 \mathcal{E}} \frac{|f(y)|}{\rho\left(x_{0}-y\right)^{|\alpha|}} d y\right)^{p} \\
\leq & C m^{\frac{n p}{2}} w(\mathcal{E})\left(\frac{1}{w(\mathcal{E})} \int_{\mathcal{E}}\left|b_{1}(x)-\left(b_{1}\right)_{\mathcal{E}, w}\right|^{2 p} w(x) d x\right)^{\frac{1}{2}} \\
& \times\left(\frac{1}{w(\mathcal{E})} \int_{\mathcal{E}}\left|b_{2}(x)-\left(b_{2}\right)_{\mathcal{E}, w}\right|^{2 p} w(x) d x\right)^{\frac{1}{2}} \\
& \times\left(\sum_{j=1}^{\infty} \int_{2^{j+1} \mathcal{E} \backslash 2 \mathcal{E}_{\mathcal{E}}} \frac{|f(y)|}{\left.\rho\left(x_{0}-y\right)^{|\alpha|} d y\right)^{p}}\right. \\
\leq & C m^{\frac{n p}{2}}\left\|b_{1}\right\|_{*}^{p}\left\|b_{2}\right\|_{*}^{p} w(\mathcal{E})\left(\sum_{j=1}^{\infty} \frac{1}{\left|2^{j \mathcal{E}}\right|}\left(\int_{2^{j+1} \mathcal{E}}|f(y)|^{p} w(y) d y\right)^{\frac{1}{p}}\right. \\
& \left.\times\left(\int_{2^{j+1} \mathcal{E}} w(y)^{-\frac{p^{\prime}}{p}} d x\right)^{\frac{1}{p^{\prime}}}\right)^{p},
\end{aligned}
$$

since $w \in A_{p}$, and by the definition of a weighted Morrey space, we get

$$
\begin{aligned}
\int_{\mathcal{E}}\left|I I_{1}(x)\right|^{p} w(x) d x & \leq C m^{\frac{n p}{2}}\|\vec{b}\|^{p} w(\mathcal{E})\left(\sum_{j=1}^{\infty} w\left(2^{j+1} \mathcal{E}\right)^{\frac{-1}{p}}\left(\int_{\mathcal{2}^{j+1} \mathcal{E}}|f(y)|^{p} w(y) d y\right)^{\frac{1}{p}}\right)^{p} \\
& \leq C m^{\frac{n p}{2}}\|\vec{b}\|^{p} w(\mathcal{E})\left(\sum_{j=1}^{\infty} w\left(2^{j+1} \mathcal{E}\right)^{\frac{\kappa-1}{p}}\|f\|_{L^{p, \kappa}(w)}\right)^{p} \\
& \leq C m^{\frac{n p}{2}}\|\vec{b}\|^{p} w(\mathcal{E})\left(\sum_{j=1}^{\infty} D^{j \frac{\kappa-1}{p}} w(\mathcal{E})^{\frac{\kappa-1}{p}}\|f\|_{L^{p, \kappa}(w)}\right)^{p} \\
& \leq C m^{\frac{n p}{2}}\|\vec{b}\|^{p} w(\mathcal{E})^{\kappa}\|f\|_{L^{p, \kappa}(w)}^{p}
\end{aligned}
$$

The third inequality is obtained by Lemma A. 
For $I_{2}(x)$, note that $\lambda_{i}=\left(b_{i}\right)_{\mathcal{E}, w}=\frac{1}{w(\mathcal{E})} \int_{\mathcal{E}} b_{i}(x) w(x) d x, i=1,2$. By Hölder's inequality and $\rho\left(x_{0}-y\right) \leq C \rho(x-y)$, we get

$$
\begin{aligned}
\int_{\mathcal{E}}\left|I I_{2}(x)\right|^{p} w(x) d x \leq & C m^{\frac{n p}{2}} w(\mathcal{E})\left(\int_{\mathbb{R}^{n} \backslash \mathcal{E}} \frac{\left|\left(b_{1}(y)-\left(b_{1}\right)_{\mathcal{E}, w}\right)\left(b_{2}(y)-\left(b_{2}\right)_{\mathcal{E}, w}\right)\right|}{\rho\left(x_{0}-y\right)^{|\alpha|}}|f(y)| d y\right)^{p} \\
\leq & C m^{\frac{n p}{2}} w(\mathcal{E})\left(\sum_{j=1}^{\infty} \frac{1}{|2 j \mathcal{E}|} \int_{2^{j+1} \mathcal{E} \backslash 2 j \mathcal{E} \mathcal{E}} \mid\left(b_{1}(y)-\left(b_{1}\right)_{\mathcal{E}, w}\right)\right. \\
& \left.\times\left(b_{2}(y)-\left(b_{2}\right)_{\mathcal{E}, w}\right)|f(y)| d y\right)^{p} \\
\leq & C m^{\frac{n p}{2}} w(\mathcal{E})\left(\sum_{j=1}^{\infty} \frac{1}{|2 j \mathcal{E}|}\left(\int_{2^{j+1} \mathcal{E}}|f(y)|^{p} w(y) d y\right)^{\frac{1}{p}}\right. \\
& \times\left(\int_{2^{j+1} \mathcal{E}}\left|\left(b_{1}(y)-\left(b_{1}\right)_{\mathcal{E}, w}\right)\right|^{2 p^{\prime}} w(y)^{-\frac{p^{\prime}}{p}} d y\right)^{\frac{1}{2 p^{\prime}}} \\
& \left.\times\left(\int_{2^{j+1} \mathcal{E}}\left|\left(b_{2}(y)-\left(b_{2}\right)_{\mathcal{E}, w}\right)\right|^{2 p^{\prime}} w(y)^{-\frac{p^{\prime}}{p}} d y\right)^{\frac{1}{2 p^{\prime}}}\right)^{p} .
\end{aligned}
$$

Indeed, by Lemma $B$ we know $B M O\left(\mathbb{R}^{n}\right)$ is equivalent to $B M O(w), w \in A_{\infty}$. Let $W=w^{-\frac{p^{\prime}}{p}} \in$ $A_{p^{\prime}} \subset A_{\infty}, b_{i} \in B M O\left(\mathbb{R}^{n}\right), i=1,2$. For any ellipsoid $\mathcal{E}$, by using Lemma $\mathrm{B}$ and Lemma $\mathrm{C}$, we show

$$
\left(\frac{1}{W\left(2^{j+1} \mathcal{E}\right)} \int_{2^{j+1} \mathcal{E}}\left|b_{i}(y)-\left(b_{i}\right)_{\mathcal{E}, w}\right|^{2 p^{\prime}} W(y) d y\right)^{\frac{1}{2 p^{\prime}}} \leq C j\left\|b_{i}\right\|_{*} .
$$

Thus, since $w \in A_{p}$, it yields

$$
\begin{aligned}
\int_{\mathcal{E}}\left|I I_{2}(x)\right|^{p} w(x) d x \leq & C m^{\frac{n p}{2}} w(\mathcal{E})\left(\sum_{j=1}^{\infty} \frac{j^{2}}{|2 j \mathcal{E}|}\left\|b_{1}\right\|_{*}\left\|b_{2}\right\|_{*} W\left(2^{j+1} \mathcal{E}\right)^{\frac{1}{p^{\prime}}}\right. \\
& \left.\times\left(\int_{2^{j+1} \mathcal{E}}|f(y)|^{p} w(y) d y\right)^{\frac{1}{p}}\right)^{p} \\
\leq & C m^{\frac{n p}{2}} w(\mathcal{E})\|\vec{b}\|^{p}\|f\|_{L^{p, \kappa}(w)}^{p}\left(\sum_{j=1}^{\infty} \frac{j^{2}}{w\left(2^{j+1} \mathcal{E}\right)^{\frac{1-\kappa}{p}}}\right)^{p} \\
\leq & C m^{\frac{n p}{2}} w(\mathcal{E})^{\kappa}\|\vec{b}\|^{p}\|f\|_{L^{p, \kappa}(w)}^{p} .
\end{aligned}
$$

The last inequality is obtained by Lemma A and the D'Alembert judge method of positive series.

For $I I_{3}(x)$, by the inequality $\rho\left(x_{0}-y\right) \leq C \rho(x-y)$ since $w \in A_{p} \subset A_{\infty}$, by Lemma B, we have

$$
\begin{aligned}
& \int_{\mathcal{E}}\left|I I_{3}(x)\right|^{p} w(x) d x \\
& \quad \leq C m^{\frac{n p}{2}} \int_{\mathcal{E}}\left|\left(b_{1}(x)-\lambda_{1}\right) \int_{\mathbb{R}^{n} \backslash 2 \mathcal{E}} \frac{\left|b_{2}(y)-\lambda_{2}\right|}{\rho(x-y)^{|\alpha|}}\right| f(y)|d y|^{p} w(x) d x
\end{aligned}
$$




$$
\begin{aligned}
& \leq C m^{\frac{n p}{2}} \int_{\mathcal{E}}\left|\left(b_{1}(x)-\lambda_{1}\right)\right|^{p} w(x) d x\left(\int_{\mathbb{R}^{n} \backslash 2 \mathcal{E}} \frac{\left|b_{2}(y)-\lambda_{2}\right|}{\rho\left(x_{0}-y\right)^{|\alpha|}}|f(y)| d y\right)^{p} \\
& \leq C m^{\frac{n p}{2}} w(\mathcal{E})\left\|b_{1}\right\|_{*}^{p}\left(\int_{\mathbb{R}^{n} \backslash 2 \mathcal{E}} \frac{\left|b_{2}(y)-\lambda_{2}\right|}{\rho\left(x_{0}-y\right)^{|\alpha|}}|f(y)| d y\right)^{p} .
\end{aligned}
$$

By Hölder's inequality, Lemma B and Lemma C, we get

$$
\begin{aligned}
\int_{\mathbb{R}^{n} \backslash 2 \mathcal{E}} \frac{\left|b_{2}(y)-\lambda_{2}\right|}{\rho\left(x_{0}-y\right)^{|\alpha|}|f(y)| d y \leq} & C \sum_{j=1}^{\infty} \frac{1}{\left|2^{j \mathcal{E}}\right|} \int_{2^{j+1} \mathcal{E}}\left|b_{2}(y)-\lambda_{2}\right| f(y) \mid d y \\
\leq & C \sum_{j=1}^{\infty} \frac{1}{\left|2^{j} \mathcal{E}\right|}\left(\int_{j^{j+1} \mathcal{E}}|f(y)|^{p} w(y) d y\right)^{\frac{1}{p}} \\
& \times\left(\int_{2^{j+1} \mathcal{E}}\left|b_{2}(y)-\lambda_{2}\right|^{p^{\prime}} w(y)^{-\frac{p^{\prime}}{p}} d y\right)^{\frac{1}{p^{\prime}}} \\
\leq & C\left\|b_{2}\right\|_{*} \mid f \|_{L^{p, \kappa}(w)} \sum_{j=1}^{\infty} j \frac{w\left(2^{j+1} \mathcal{E}\right)^{\frac{\kappa}{p}}}{\left|2^{j} \mathcal{E}\right|}\left(\int_{2^{j+1} \mathcal{E}} w(y)^{-\frac{p^{\prime}}{p}} d y\right)^{\frac{1}{p^{\prime}}} \\
\leq & C\left\|b_{2}\right\|_{*}\|f\|_{L^{p, \kappa}(w)} \sum_{j=1}^{\infty} \frac{j}{w\left(2^{j+1} \mathcal{E}\right)^{\frac{1-\kappa}{p}}}
\end{aligned}
$$

indeed for $0<\kappa<1$, by using Lemma A, we have that

$$
\sum_{j=1}^{\infty} \frac{j}{w\left(2^{j+1} \mathcal{E}\right)^{\frac{1-\kappa}{p}}} \leq \sum_{j=1}^{\infty} \frac{j}{D^{(j+1) \frac{1-\kappa}{p}}} w(\mathcal{E})^{\frac{\kappa-1}{p}} \leq C w(\mathcal{E})^{\frac{\kappa-1}{p}}
$$

Thus, we conclude

$$
\int_{\mathcal{E}}\left|I I_{3}(x)\right|^{p} w(x) d x \leq C m^{\frac{n p}{2}} w(\mathcal{E})^{\kappa}\|\vec{b}\|^{p}\|f\|_{L^{p, \kappa}(w)}^{p} .
$$

In the same way, we shall get the result of $I I_{4}(x)$

$$
\int_{\mathcal{E}}\left|I I_{4}(x)\right|^{p} w(x) d x \leq C m^{\frac{n p}{2}} w(\mathcal{E})^{\kappa}\|\vec{b}\|^{p}\|f\|_{L^{p, \kappa}(w)}^{p} .
$$

Which together with (3.1)-(3.7), for $1<p<\infty$, the proof of Theorem 1.2 is finished.

Now, we are in a position to consider the case $p=1$. In general, the singularity of the commutator is stronger than the singular integral, and the endpoint case $p=1$ of the commutator is not even obtained. Thus, the result for the case $p=1$ of the multilinear commutator is interesting. We split $f$ as above by $f=f_{1}+f_{2}$, which yields

$$
\begin{aligned}
\lambda w(\{x \in \mathcal{E}:|[\vec{b}, T] f(x)|>\lambda\}) \leq & C \sum_{s, m}\left\|b_{s m}\right\|_{L^{\infty}} \lambda w\left(\left\{x \in \mathcal{E}:\left|\left[\vec{b}, T_{s m}\right] f(x)\right|>\lambda\right\}\right) \\
\leq & C \sum_{s, m} m^{-2 l}\left[\lambda w\left(\left\{x \in \mathcal{E}:\left|\left[\vec{b}, T_{s m}\right] f_{1}(x)\right|>\lambda / 2\right\}\right)\right. \\
& \left.+\lambda w\left(\left\{x \in \mathcal{E}:\left|\left[\vec{b}, T_{s m}\right] f_{2}(x)\right|>\lambda / 2\right\}\right)\right] \\
= & C \sum_{s, m} m^{-2 l}[I I I+I V]
\end{aligned}
$$


for any ellipsoid $\mathcal{E}, \lambda>0$ and integer $l>0$. For the term $I I I$, we use Lemma G. It follows that

$$
\begin{aligned}
I I I & \leq C \int_{\mathbb{R}^{n}} \Phi\left(\left|f_{1}\right|\right)(x) w(x) d x \\
& \leq C w(\mathcal{E})^{\kappa}\|f\|_{L^{\Phi, \kappa}(w)} .
\end{aligned}
$$

For the last term $I V$, without loss of generality, we still suppose $N=2$. By homogeneity, it is enough to assume $\lambda / 2=\left\|b_{1}\right\|_{*}=\left\|b_{2}\right\|_{*}=1$, and hence we only need to prove

$$
w\left(\left\{x \in \mathcal{E}:\left|\left[\vec{b}, T_{s m}\right] f_{2}(x)\right|>1\right\}\right) \leq C w(\mathcal{E})^{\kappa}\|f\|_{L^{\Phi, \kappa}(w)} .
$$

In fact, by Lemma F, we get

$$
\begin{aligned}
w\left(\left\{x \in \mathcal{E}:\left|\left[\vec{b}, T_{s m}\right] f_{2}(x)\right|>1\right\}\right) & \leq \sup _{t>0} \frac{1}{\Phi\left(\frac{1}{t}\right)} w\left(\left\{x \in \mathcal{E}:\left|\left[\vec{b}, T_{s m}\right] f_{2}(x)\right|>t\right\}\right) \\
& \leq C \sup _{t>0} \frac{1}{\Phi\left(\frac{1}{t}\right)} w\left(\left\{x \in \mathcal{E}: M_{\Phi} f_{2}(x)>t\right\}\right) \\
& =C \sup _{t>0} \frac{1}{\Phi\left(\frac{1}{t}\right)} w\left(\left\{x \in \mathcal{E}: M\left(\Phi\left|f_{2}\right|\right)(x)>t\right\}\right),
\end{aligned}
$$

where $\Phi(t)=t \log ^{N}(e+t)$. We use the Fefferman-Stein maximal inequality

$$
\int_{x: M f(x)>t} \phi(t) d x \leq \frac{C}{t} \int_{\mathbb{R}^{n}}|f(x)| M \phi(x) d x,
$$

for any functions $f$ and $\phi \geq 0$. This yields

$$
\begin{aligned}
w\left(\left\{x \in \mathcal{E}: M\left(\Phi\left|f_{2}\right|\right)(x)>t\right\}\right) & \leq \frac{1}{t} \int_{\left\{x \in \mathbb{R}^{n}: M\left(\Phi\left|f_{2}\right|\right)(x)>t\right\}} \chi_{\mathcal{E}}(x) w(x) d x \\
& \leq \frac{C}{t} \int_{\mathbb{R}^{n}} \Phi\left(\left|f_{2}\right|\right)(x) M\left(w \chi_{\mathcal{E}}\right)(x) d x \\
& =\frac{C}{t}\left(\int_{3 \mathcal{E}}+\int_{\mathbb{R}^{n} \backslash \mathcal{E}}\right) \Phi\left(\left|f_{2}\right|\right)(x) M\left(w \chi_{\mathcal{E}}\right)(x) d x \\
& =\frac{C}{t}\left(I V_{1}+I V_{2}\right) .
\end{aligned}
$$

For $I V_{1}$, since $w \in A_{1}$, it follows that

$$
\begin{aligned}
I V_{1} & \leq C \int_{3 \mathcal{E}} \Phi(|f|)(x) w(x) d x \\
& \leq C w(3 \mathcal{E})^{\kappa}\|\Phi(|f|)\|_{L^{1, \kappa}(w)} \\
& \leq C w(\mathcal{E})^{\kappa}\|f\|_{L^{\Phi, \kappa}} .
\end{aligned}
$$

To estimate the term $I V_{2}$, we first consider the form

$$
\frac{1}{|\mathcal{F}|} \int_{\mathcal{E} \cap \mathcal{F}} w(y) d y
$$


for any $x \in \mathbb{R}^{n} \backslash 3 \mathcal{E}, x \in \mathcal{F}$ and $\mathcal{F} \cap \mathcal{E} \neq \emptyset$. By simple geometric observation, we have

$$
\begin{aligned}
\frac{1}{|\mathcal{F}|} \int_{\mathcal{E} \cap \mathcal{F}} w(y) d y & \leq C \frac{1}{\rho\left(x-x_{0}\right)^{|\alpha|}} \int_{\mathcal{E}} w(y) d y \\
& =\frac{C}{\rho\left(x-x_{0}\right)^{|\alpha|}} w(\mathcal{E}) .
\end{aligned}
$$

Therefore, we obtain

$$
M\left(w \chi_{\mathcal{E}}\right)(x) \leq \frac{C}{\rho\left(x-x_{0}\right)^{|\alpha|}} w(\mathcal{E})
$$

Since $w \in A_{1}$ satisfies the doubling condition and Lemma A, we estimate the term $I V_{2}$ as follows:

$$
\begin{aligned}
I V_{2} & \leq C \int_{\mathbb{R}^{n} \backslash 3 \mathcal{E}} \frac{\Phi(|f|)(x)}{\rho\left(x-x_{0}\right)^{|\alpha|}} w(\mathcal{E}) d x \\
& \leq C w(\mathcal{E}) \sum_{j=1}^{\infty} \frac{1}{\left|3^{j} \mathcal{E}\right|} \int_{3^{j+1} \mathcal{E}} \Phi(|f|)(x) d x \\
& \leq C w(\mathcal{E})\|\Phi(|f|)\|_{L^{1, \kappa}(w)} \sum_{j=1}^{\infty} \frac{1}{w\left(3^{j} \mathcal{E}\right)^{1-\kappa}} \\
& \leq C w(\mathcal{E})^{\kappa}\|f\|_{L^{\Phi, \kappa}} .
\end{aligned}
$$

The last inequality is similar to (3.4). Noting that $t \Phi\left(\frac{1}{t}\right)>1$, from (3.8)-(3.11), we conclude

$$
w\left(\left\{x \in \mathcal{E}:\left|\left[\vec{b}, T_{s m}\right] f_{2}(x)\right|>1\right\}\right) \leq C w(\mathcal{E})^{\kappa}\|f\|_{L^{\Phi, \kappa}(w)} .
$$

Thus, the proof of Theorem 1.2 is completed.

\section{Competing interests}

The authors declare that they have no competing interests.

\section{Authors' contributions}

XFY conceived of the study and drafted the manuscript. XSZ participated in the discussion. All authors read and approved the final manuscript.

\section{Acknowledgements}

XFY research was partially supported by the National Natural Sciences Foundation of China (10961015; 11161021). XSZ research was supported by the National Natural Sciences Foundation of China (11161021).

Received: 24 August 2012 Accepted: 5 November 2012 Published: 17 December 2012

\section{References}

1. Fabes, EB, Rieviève, N: Singular integrals with mixed homogeneity. Stud. Math. 27, 19-38 (1966)

2. Jones, BF: On a class of singular integrals. Am. J. Math. 86, 441-462 (1964)

3. Softova, L: Singular integrals and commutators in generalized Morrey spaces. Acta Math. Sin. 22(3), 757-766 (2006)

4. Pérez, C, Trujillo-González, R: Sharp weighted estimates for multilinear commutators. J. Lond. Math. Soc. 65, 672-692 (2002)

5. Xu, JS: Multilinear commutators of singular integrals in generalized Morrey spaces. Chin. J. Contemp. Math. 27(1), 83-92 (2006)

6. Komori, Y, Shirai, S: Weighted Morrey spaces and a singular integral operator. Math. Nachr. 282(2), $219-231$ (2009)

7. Wang, H: Some estimates for Bochner-Riesz operators on the weighted Morrey spaces. arXiv:1011.1622v1 [math. CA] (2010) 
8. Wang, $\mathrm{H}$ : The boundedness of some operators with rough kernel on the weighted Morrey spaces. arXiv:1011.5763v1 [math. CA] (2010)

9. Wang, $\mathrm{H}$ : The boundedness of fractional integral operators with rough kernels on the weighted Morrey spaces. arXiv:1203.1441v1 [math. CA] (2012)

10. He, S: The boundedness of some multilinear operator with rough kernel on the weighted Morrey spaces. arXiv:1111.5463v3 [math. CA] (2012)

11. John, F, Nirenberg, L: On functions of bounded mean oscillation. Commun. Pure Appl. Math. 14, 415-426 (1961)

12. Garcia-Cuerva, J, Rubio de Francia, J: Weighted Norm Inequalities and Related Topics. North-Holland, Amsterdam (1985)

13. Muckenhoupt, B, Wheeden, R: Weighted bounded mean oscillation and the Hilbert transform. Stud. Math. 54, 221-237 (1976)

14. Duoandikoetxea, J: Fourier Analysis. Am. Math. Soc., Providence (2000)

15. Calderón, AP, Zygmund, A: Singular integral operators and differential equations. Am. J. Math. 79(4), 901-921 (1957)

16. Chiarenza, F, Frasca, M, Longo, P: $W^{2, P}$-solvability of the Dirichlet problem for nondivergence elliptic equations with VMO coefficients. Trans. Am. Math. Soc. 334, 841-853 (1993)

doi:10.1186/1029-242X-2012-302

Cite this article as: Ye and Zhu: Estimates of singular integrals and multilinear commutators in weighted Morrey spaces. Journal of Inequalities and Applications 2012 2012:302.

\section{Submit your manuscript to a SpringerOpen ${ }^{\ominus}$ journal and benefit from:}

- Convenient online submission

Rigorous peer review

Immediate publication on acceptance

Open access: articles freely available online

- High visibility within the field

- Retaining the copyright to your article 